\title{
MOULTON ON DIFFERENTIAL EQUATIONS
}

Differential Equations. By Forest Ray Moulton. New York, Macmillan Company, 1930.

This is an important book dealing exclusively with ordinary differential equations. It could have been written only by one thoroughly familiar with the details of certain phases of mathematical physics. In fact it has a decided astronomical slant. One needs only to run over the chapter headings to be convinced of this, and a careful reading of the book confirms this preliminary conviction. It is different from any other text on the subject known to the reviewer by virtue of the happy combination it achieves of theory and applications.

Various important points of the theory are illustrated by the working out in detail of the problems of elliptic motion, the deviations of falling bodies, and the damped gyroscope. A discussion of the sine-amplitude function also furnishes illustrative material. As the author justly remarks in his preface, these problems, in respect to difficulty, "are far beyond the type usually used for illustrations," and they "help to bring out the richness of the logical results contained in the general theorems, a richness which would not be suspected by a beginner in the field."

The fact that Chapter 11 is devoted wholly to numerical solutions is a further indication of the practical nature of the book. But this work is preceded by a new and careful discussion of the logical foundations of the numerical processes described. As to the details of the numerical work, it seems to the reviewer that the discussion is too elaborate for the general reader. There can be no question but that a certain amount of these numerical details is valuable for all students. But opinions will differ as to where the line should be drawn, and it is better to err by giving too many than too few. The chapter on Linear Differential Equations with Constant Coefficients also contains important new material, but it is also more detailed and comprehensive than is desirable for the general reader.

The main part of the book ends with a chapter on equations with periodic coefficients and one on equations in infinitely many variables. Both of these chapters contain new material.

The historical sketches at the ends of the chapters contain much important information and add materially to the value of the book. There is also a good list of questions and problems with each chapter. In addition there is a series of appendices in which a number of the standard theorems of analysis which are used in the text are proved. This will be a welcome feature to many readers of the book.

We have referred to the familiarity with the practical details shown by the author. But he has not failed to give due attention to questions of theory. In fact most of the book is devoted to a presentation of the classical theory, and the reader cannot fail to be impressed by the rigor of the demonstrations and the careful attention to clearness that is obvious on every page. He will be convinced that the author has come very close to his ideal as set forth in the 
preface- "It would be inexcusable to discuss general theorems of differential equations and not to treat them with the rigor and completeness of reasoning that are characteristic of analysis of the present day. The aim of the book has been to meet current standards of rigor in the discussion of every subject treated."

There is, of course, a full discussion of the current existence theorems. In addition, the dependence of the solutions upon parameters is handled in a very suggestive and satisfactory way, as is also the expansion of a solution in the neighborhood of a variable point.

In writing such a book as this it is obviously necessary, as the author observes, to omit many important topics. We note here for the benefit of the reader that he will find nothing in the book concerning the nature of solutions in the neighborhood of singular points and no discussion of general boundary problems or of linear differential systems.

There is nothing about the adjoint of a linear homogeneous differential operator or about Green's functions. Neither is there anything about the solution of linear equations by definite integrals or about the asymptotic expansion of solutions.

The number of misprints seems to be small. We list here a few that have come to notice. On page 124 the coefficient $a_{5}$ should also be subject to the condition of being a real constant. The singular point referred to on line 4 of $\S 75$, page 125 , should be $\pm \frac{1}{2}(-1)^{1 / 2} P_{2}$. The equation $t=T$ on line 23 of page 191 should be $t-t_{0}=T$. On page 209 , line 5 from the bottom, $r / m$ should be replaced by $(r-\mu) / M$, and on page 249 , line 2 , the inequality sign should be replaced by the equality sign. The words the equations should be inserted after the word consequently on page 48, line 6 from the bottom.

Then there are certain slight inaccuracies for which the printer is not responsible. For example, on page 103 the statement is made that "the right members of equations (30) can be expressed in power series in e." But these right members are all zero. Evidently the equations in question were in another form when this statement was written, and when the equations were changed the statement was not changed accordingly. In the figure on page 142 the point $P_{3}$ should be between $Q$ and $P_{0}$. The inequalities (9) on page 227 are implied by inequalities (8), and not the latter by the former.

The author says on page 226 "Now let the process pass toward a limit by subdividing the whole interval for which $t$ and the $X_{i}{ }^{(k)}(t)$ remain in tine region $R$ into a greater and greater number of subintervals in such a way that the length of each subinterval shall approach zero." This is meaningless unless the process of subdivision is such that every subinterval preserves its identity from its first appearance on. But it is not necessary to pass toward the limit in such a particular way.

The reader now and then comes upon a statement that causes him considerable surprise. For example, on page 117 he is told that in the principal rectangle the sine-amplitude function is "real on the line $t=\frac{1}{2}(-1)^{1 / 2} P_{2} ;$ " and on page 121 that "With these initial conditions $x$ and its derivatives are real even functions of $t-P^{\prime}$." The proof on page 131 that the second of equations (45) is a consequence of the first and of the first of equations (40) is not all clear although the conclusion itself seems fairly obvious. 
The argument at the middle of page 49 shows merely that

$$
\left|\begin{array}{cccc}
\phi_{11}\left(t_{0}\right)-1 & \phi_{12}\left(t_{0}\right) & \phi_{13}\left(t_{0}\right) & \cdots \\
\phi_{21}\left(t_{0}\right) & \phi_{22}\left(t_{0}\right)-1 & \phi_{23}\left(t_{0}\right) & \cdots \\
\vdots & \vdots & \vdots & \\
\phi_{n 1}\left(t_{0}\right) & \phi_{n 2}\left(t_{0}\right) & \phi_{n 3}\left(t_{0}\right)-1 & \ldots
\end{array}\right|=0 \text {, }
$$

when not all the $A_{i}{ }^{(1)}$ 's are zero. The values $\phi_{i, i}\left(t_{0}\right)=1(i=1,2, \cdots, n)$ and $\phi_{i, j}\left(t_{0}\right)=0(i \neq j)$, given by the author, satisfy this condition, but it is not shown that there are not other possible solutions that make the determinant of the coefficients of equations (17) equal to zero.

On page 33, in his discussion of systems of equations of the first order of the form $d x_{i} / d t=f_{i}\left(x_{j} ; \mu ; t\right)$, where $\mu$ is a parameter independent of the $x_{j}$ and $t$, the author considers one set of conditions in which he supposes, among other conditions, that the functions $f_{i}\left(x_{j} ; \mu ; t\right)$ are analytic in the $x_{j}$ and $t$ uniformly with respect to $\mu$. The reader is left in uncertainty as to what the author means by uniform analyticity. If he means what one would naturally suppose, his condition seems to be stronger than it need be inasmuch as it is only the continuity of the $f_{i}\left(x_{j} ; \mu ; t\right)$ as functions of the $x_{j}$ and $t$ that his argument requires to be uniform with respect to the parameter $\mu$, in addition to ordinary analyticity.

The corollary to Theorem I on page 378 seems to imply that if the function $f\left(x_{1}, x_{2}, \cdots, x_{n} ; t\right)$ satisfies the Lipschitz condition, or possesses derivatives, then it is continuous simultaneously in the $x_{i}$ uniformly with respect to $t$. It is clear, however, that the author did not intend to imply so much, since he cites an example on the same page with this corollary of a function for which this is not true; namely, $f(x ; t)=x t /\left(x^{2}+t^{2}\right)$ when $x$ and $t$ are not both zero, and $f(0,0)=0$. This function is continuous with respect to $x$ and $t$ separately for finite values of $x$ and $t$, and has derivatives with respect to $x$ and $t$. But it is not continuous in $x$ at $x=0$ uniformly with respect to $t$, since if it were it would be continuous at $x=0, t=0$ simultaneously with respect to $x$ and $t$.

The statement near the middle of page 238 to the effect that the coefficients of equations (23) are constants in case the $\phi_{i}$ of equations (18) are constants is incorrect, as is shown by the simple example $d x / d t=(x-c) t$. Here $f(x, t)=(x-c) t$ and $\phi=c$. Hence $\partial f / \partial x=t$, which is not a constant. This error is repeated at the top of page 296. The argument which leads to equation (26) on page 239 is far from convincing, although it is easy to verify directly that this equation defines a particular solution of equations (23). It is not as obvious as the author implies on page 305 that, since the right member of equation (24) is an even function of $t, \phi(t)$ must be an even function of $t$.

But these are only a few minor spots on an otherwise brilliant sun.

W. B. Fite 